\title{
PEDRO ALFONSO Y LA ICONOGRAFÍA RELIGIOSA EUROPEA
}

\author{
José L. Viviente Mateu'
}

A Joaquín Lomba Fuente y Piluca, con afecto y los mejores deseos en su jubilación

\section{RESUMÉ}

On propose une biographie de Petrus Alfonsi de Huesca. On reproduit notre déduction mathématique de l'icône Trinitaire d'un parchemin de Chartres, du XIII' siècle, depuis la figure précabalistique de Petrus Alfonsi, qu'il a introduit dans son oeuvre Dialogue contre les juifs en l'année 1110. On présente aussi trois matérialisations de l'enlacement alterné de trois anneaux deux à deux non enlacés.

Palabras clave: Chartres, Enlacement, Gordiano, Link, Mesa frigia, Monedero malla, Petrus Alfonsi.

\section{SUMMARY}

A biography of Pedro Alfonso is proposed from the information today known. There is reproduced our mathematical deduction of the icon Trinitario of a parchment of Chartres of the XIIIth century from a figure precabalística introduced in 1110 by Pedro Alfonso of Huesca and it is presented three other materializations of the «alternated link of three rings any two not linked».

\section{INTRODUCCIÓN}

Agotada la monografía ${ }^{\circ} 18$ de la Academia de Ciencias Exactas, Físicas, Químicas y Naturales de Zaragoza, de mayo de 2001, donde se publicaba nuestro estudio sobre la escultura «Creación» del escultor inglés John Robinson, en esta publicación presentamos: $1^{\circ}$ ) una tabla con una más explícita biografía de Pedro Alfonso; $2^{\circ}$ ) una actualización de nuestra demostración de que el icono de Chartres sigue de una figura introducida en 1110 por Pedro Alfonso; y $3^{\circ}$ ) tres materializaciones del objeto matemático subyacente, una precisamente el mítico «nudo gordiano» ${ }^{2}$.

* Clasificación de 2.000 del MR: 57-03, 57M25.

1 Exprofesor de la Universidad C. de Madrid; Profesor A. de Matemáticas de la Sorbona (Paris 6) jubilado; Catedrático de Geometría $5^{\circ}$ (Diferencial) de la Universidad de Zaragoza, ya jubilado; Profesor Emérito, miembro numerario de la Real Academia de Ciencias Exactas, Físicas, Químicas y Naturales de Zaragoza, Académico correspondiente de la Académie de Sciences Inscriptions et Belles Letres de Toulouse, Caballero de las Palmas Académicas y de la Legión de.Honor.

2 La aplicación relativa al «Nudo Gordiano», a parte de habérsenos sido señalada por un colega de la Universidad de París de origen hindú, tambiến la supone un profesor de la Universidad de Ginebra., y nos fue recordada por la inesperada y "acertada" intervención (cual Alejandro Magno) de un buen amigo y destacado colega, especialista en Topología de baja dimensión, cuando terminábamos nuestra exposición en el «XX Encuentro de 


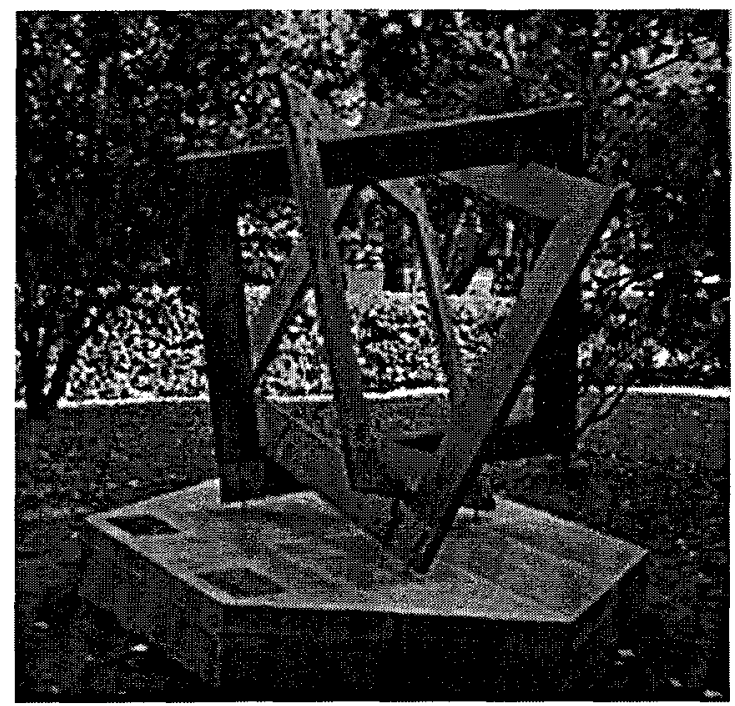

Escultura «Creación» por John Robinson instalada el 16/XI/2000 en el campus de la Universidad de Zaragoza.

La bibliografía se precisará en una próxima y más extensa publicación, la mayoría de la información se encuentra en los datos proporcionados por: la obra de J. M. Lacarra sobre el rey Alfonso I el Batallador, las dos publicaciones del Instituto de Estudios Altoaragoneses en 1996 con motivo del 900 aniversario de la conquista de Huesca por el rey Pedro I de Aragón; la obra de Tolan sobre Pedro Alfonso de 1993; la obra de Pedro Alfonso Disciplina Clericalis (traducción de E. Ducay e introducción de Ma J. Lacarra, de 1982); una de las obras de G. Scholem y la de David Gonzalo Maeso con introducción de $\mathrm{M}^{\mathrm{a}}$ Encarnación Varela Moreno.

Una de las razones de nuestro estudio de 2001 se hallaba en saber si fue en el Centro Escolar de Chartres en donde surgiera nuestro enlace, aunque no se publicase hasta el siglo XIII. Puesto que no hay ninguna otra referencia, lo intuitivo de su deducción y elegancia de su forma, nos dice que su obtención en tal Centro debió realizarse cuando Pedro Alfonso pasó por el mismo al regresar de Inglaterra hacia 1119. La expresión dada por cuatro circunferencias ya la conocía, pues debió deducirla cuando, de camino hacia el monasterio de Cluny, pasase por el Monasterio de Ripoll - lugar en el que ya en el siglo IX existía una buena biblioteca matemática con obras de Apolonio y otros matemáticos griegos-. En Cluny, además de hacer buen número de copias de su obra apologética «Diálogo contra los judios», se encargarían de distribuirlas entre los más importantes monasterios europeos. Ello justificaría la afirmación de Tolan sobre el gran número de ejemplares que del «Diálogo contra los judios» existían ya en siglo XII. En esta obra, tenemos la más antigua referencia figural de la que se deduce el icono

Topología Algebraica» realizado en Jaca del 2 al 4 de mayo de 2002. Y es que, en efecto, ante un amplio auditorio, la más rápida y fácil demostración de que el trípode soporte plegable de la mesa que presentamos (de origen, parece ser, frigio, irakí o paquistaní, posiblemente traído a la península Ibérica por los árabes), es una materiàlización del «enlace matemático alternado de tres anillos dos a dos no enlazados», sigue al cortar (o romper) uno de los «aros» por un corte del contorno del orificio central. De ser una tal materialización, la rotura de uno de los «aros» descompone el trípode en tres cuerpos iguales independientes, topológicamente equivalentes a un «donut» o anillo material. (uno de ellos roto, claro, pero que el citado profesor especialista, el pasado 3/V/2002, al final de nuestra conferencia en Jaca, se apresuró a recomponer y superponer sobre los otros dos, demostrando su igualdad). 
de Chartres, hecho que, junto a las propiedades matemáticas últimamente descubiertas en la Facultad. de Ciencias de Zaragoza, nos induce a denominarlo «enlace de Aragón». Es en el Título VI de esta obra apologética, donde Pedro dice a su alter ego Moisés:

La trinidad es algo sutil e inefable, difícil de explicar, de la cual los profetas no han hablado sino oscura y veladamente. Mas cuando vino Cristo, que es una de las tres personas, la reveló a la mente de los fieles sólo en relación con su capacidad. Pero si te fijas minuciosamente en cómo está explicado el nombre de Dios en el Secreta Secretorum, ese nombre, digo, se escribe con tres letras aunque los signos sean cuatro pues uno de ellos está repetido; si lo miras bien, repito, verás que el nombre mismo es uno y tres: uno por la unidad que significa, tres porque se refiere a la existencia trina de personas, pues cons-

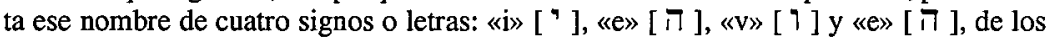
cuales el primero y el segundo, es decir: «i» ['] más «e» [ $\mathrm{A}]$, constituyen un nombre. Lo mismo sucede si unes la segunda letra y la tercera, es decir, «e» [ $\mathrm{i}] \mathrm{y}$ « $\mathrm{V}$ » [ 1 ], y así tienes otro nombre. Igualmente si unes sólo la tercera y la cuarta, es decir, «V» [ 1 ] y «e» $[\pi]$ ], obtendrás el tercer nombre. Y si miras todos esos signos o letras en su orden no hallarás sino un solo nombre, como se ve en la figura 1.

La figura 1, en la tesis de Mieth está tomada del manuscrito $\mathrm{n}^{\circ} 5080$ (f.185 vo) de la Biblioteca Nacional de París. Figura de la que E. Ducay afirma es «Signo cabalístico de la Trinidad. Las letras son Yod, Hei, Vau y Hei, YHWH. Hei y Vau aparecen raras veces en el Talmud como abreviatura del nombre de Dios». Si se sigue a Tolan, en el comentario a la citada tesis, se lee: «que muestra como IEVE es el nombre del Dios único compuesto de los nombres de las personas de la Trinidad: IE, EV y VE». Tolan afirma que tal hecho es una aportación original de Pedro Alfonso, si bien debemos añadir que su motivación la obtendría del Sefer Yesirah (Libro de la Creación, también llamado del Conocimiento) obra del siglo VI o VII al que sin duda se refiere cuando habla del Secreta Secretorum.

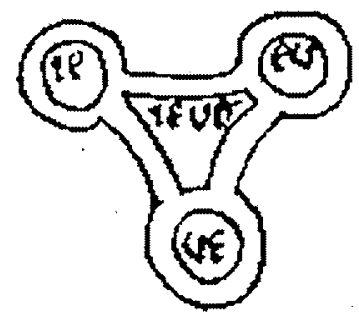

Figura 1

Pero veamos ya la posible biografía de Pedro Alfonso, que presentamos con las circunstancias culturales, religiosas, políticas y sociales más destacadas de la época en que vivió.

\section{UNA BIOGRAFÍA DE PEDRO ALFONSO DE HUESCA}

\section{Vida y obra}

1071 Nace en Huesca, en ambiente de lucha entre árabes y cristianos, un judío que se llamó Moisés Sefardí. Estudió árabe, ciencia, filosofía y el Corán con ideas del misticismo sufí. En familia aprendió hebreo, leyó la Torá y fue atraído por el secretismo y esoterismo del Talmud. De facilidad de palabra, busca la verdad, con gran capacidad de es- 
peculación y crítica racionalista; pronto creyó ver contradicciones en el Talmud, a la vez que vería la piedad de los mozárabes, en aquel momento deprimidos por la muerte de su obispo Sancho.

1087 Conocido por el visir de Ahmed II le facilita el acceso a la biblioteca de la Alfajería y le orienta en sus estudios de astronomía, astrología y medicina. Su atracción hacia la mística se racionaliza cada vez más por su superior formación. Lee la Merkabah, el Sefer Yesirah (con los comentarios de Saadia Gaon), y la obra Enciclopedia de los Hermanos de la Pureza, viéndose inducido a seguir una formación rabínica, primero en la aljama de Zaragoza y el año:

1093 en la de Huesca. En su sinagoga inicia la actividad lectora y de plática, lo que le daría la popularidad y fama que atraería la atención del obispo Esteban. En este tiempo conocería a Abraham bar Hiyya que le daría alguna de sus obras sobre astronomía.

1097 Establecida la capital de Aragón en Huesca, Moseh Sefardí pasa a ser médico de Alfonso y auxiliar del obispo Esteban en su función de maestro y asesor del futuro rey. M. Sefardí vería que sus reparos al Talmud lo eran también para el preceptor del Rey (y canónigo de Huesca desde 1104) Pedro de Almería, quien ayudaría a M. Sefardí en su catequesis por los monjes del monasterio de San Juan de la Peña (a donde se retiró después del bautizo de Pedro Alfonso).

1106 El obispo Esteban bautiza a Pedro Alfonso siendo padrino el rey Alfonso I. Ante los ataques que a Pedro Alfonso hicieron sus antiguos correligionarios por su conversión, Pedro Alfonso respondió con la obra apologética «Diálogo contra los judios» escrita entre 1106 y 1109. Obra que en su Título VI está la figura que reproducimos en la página 31.

1109 Los monjes aconsejarían que llevase su obra al Monasterio de Ripoll $y$, allí (o en el centro escolar de Vic) se introduciría la figura que $\mathrm{E}$. Ducay dice es de mayor uso (y que indujo a error a Joaquín de Fiore):

Y precisamente en Ripoll, a finales de 1109, sería donde Pedro Alfonso añadiría el título «Imperator Hispaniae» en su alusión a $\mathrm{Al}$ fonso I, obtenido por su malogrado matrimonio con Urraca hija del

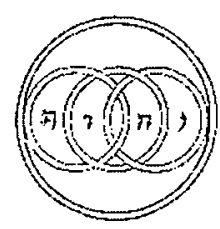
rey de Casilla y León.

Los monjes benedictinos de Ripoll, ante el valor de la obra y en interés de su mayor y más pronta divulgación, le remitirían a Cluny, el más impiportante monasterio de la cristiandad en aquel momento, con Pedro el Venerable como abad.

1110 En Cluny además de realizar las copias, procederían a enviarlas a los monasterios y centros catedralicios más importantes de Europa. Pero como conocen las dificultades que atraviesan en Francia los iniciadores de la escolástica (abad de Bec, Pedro Abelardo, etc.) por defender un tipo de docencia no hierática, aconsejan a Pedro Alfonso que pase a Inglaterra. Allí el obispo de Canterbury, además de introducirlo en la corte y posibilitar que el rey Enrique I «el Clérigo» lo tome como su médico, le facilita la práctica de la enseñanza del cuadrivium con la nueva didáctica propuesta por Pedro Alfonso.

1115 Alumno de aquella época fue el lorenés Walcher, prior del monasterio de Malvern. Quizás lo fuese también Adelardo de Bath (del que se conservan unas Tablas Astronómicas en latín publicadas en 1125) ya que defendió, como Pedro Alfonso, el uso de la razón para alcanzar la verdad. Además, ya en 1116 Pedro Alfonso habíapưblicado en latín unas Tablas Astronómicas (de las que quedan unos tres capítulos, la introducción y un prólogo) basadas en las de al-Jwarizmi/Maslama. El desafío al hieratismo docente galo de Adelardo Bath tiene también su precedente en el escrito de Pedro Alfonso titulado Carta a los estudiosos franceses.

1120 De regreso a Aragón, debió detenerse algún mes en la Escuela de Chartres donde habrían recibido una copia del Diálogo contra los judios, lo que justificaría fuese el primer centro francés en poner en práctica su innovador método docente. Todo hace pensar que fue 
en este encuentro cuando, discutiendo sobre su símbolo de la Trinidad, se obtendría el «icono de Chartres» publicado en el siglo XIII. También fue la ocasión de que Pedro Alfonso conociera el infortunio de Pedro Abelardo y su fiel y enamorada Eloisa.

1121 Pedro Alfonso está en Zaragoza. Pedro Alfonso, ante las circunstancias que había conocido durante su estancia en Francia e Inglaterra, al regresar a Zaragoza, opta por el anonimato.

1122 Se casa con una judía de origen tudelano, se acoge a la protección que Alfonso I acababa de conceder a los judíos de Tudela, que había sido repoblada por Normandos, y allí fija su residencia.

En Tudela no le ha de faltar trabajo como médico, como docente e, incluso, como un colaborador del «grupo de traductores» que el obispo Miguel de Tarazona ha creado en su diócesis (Miguel debió conocer su actividad por sí mismo o por el obispo Esteban). Muy probablemente alguna de las múltiples traducciones al latín que en aquella época se hicieron debieron ser obra de Pedro Alfonso, particularmente las del

Sefer Yesiráh y comentarios de Saadia Gaon.

Al menos tuvo un hijo, Don Belasco, y consciente de que por sus años no podría ser su consejero, se puso a escribir y nos dejó a todos su célebre tratado de moral Disciplina Clericalis. Murio después de $\mathbf{1 1 4 2 .}$

\section{Contexto cultural y religioso}

Como señala Tolan, la situación la enmarca un cristianismo cada día más pujante, un islam más combativo y un judaísmo enfrentado a la filosofía griega y a sus divisiones internas.

1065 Nace en Barcelona Abraham bar Hiyya. En su obra el Libro del Revelador y Meditación del Alma cita la conversión de Moseh Sefardí.

Gran desarrollo cultural de la taifa de Zaragoza con obras científicas por al-Muqtadir, al-Mutamin y su visir (judío converso al islamismo) que estudia la física de Aristóteles, y escribió en árabe y hebreo obras de geometría, astronomía y lógica.

1071 Ramiro I culmina la obra de restauración monástica de Benito Aniano en San Juan de la Peña, nuevo centro religioso que reemplaza al de Siresa.

1075 Nace en Tudela Yehuda Sam.. Ha Levi, médico, filósofo, poeta autor de El Kuzari.

1083 Muere el obispo Sancho y se deja sin cubrir el obispado mozárabe de Huesca.

1085 Nace Ibn Bayya (+1139) dicho Avempace, médico, astrónomo, matemático y poeta. Introductor de la filosofía aristotélica que llevó a Europa Averroes. El misticismo sufí lo interpretó en un misticismo intelectual. Primer pensador, musulmán y cristiano, que desarrolla la idea del sabio solitario.

1085 Nace Abraham ben 'Ezra' (+1164), escribió sobre exégesis bíblica, obras de filosofía, gramática, poesía, matemáticas, astronomía, física, cosmología y astrología. En total más de 180 obras. Fue traductor del árabe al griego y un gran viajero difundiendo sus conocimientos

1093 Se nombra arzobispo de Canterbury al escolástico Anselmo de Aosta abad de Bec (Normandía). Partidario de alcanzar la fe por la razón. Apoyó la tesis de Gregorio VII de supeditar el poder secular a la Iglesia.

1098 Roberto de Molesmes funda la Orden del Cister, orden más ascética que Cluny, con la famosa Carta de la Caridad de Harding, propagada por San Bernardo (de Claravall).

1100 A causa de una crisis religiosa personal, el filósofo árabe al-Gazali busca como sufí la verdad en la fe lejos de su familia y de la docencia.

1100 Muere San Bruno fundador de la Orden de los Cartujos, surgida a consecuencia de la reforma gregoriana. 
1109 Yehuda ben Samuel ha-Levi huye de Toledo hacia zona musulmana al ser asesinado el judío Aben Ferrusel.

1111 Muere al-Gazali, teólogo y filósofo árabe autor de la obra Destrucción de los filósofos.

1111 Bernardo de Claravall entra en la Orden del Cister.

1118 Conquistada Alcalá de Henares, el Arzobispo de Toledo decreta la igualdad de judíos y cristianos. Avempace huye de Zaragoza.

1118 Se designa San Olegario arzobispo deTarragona, enlace entre Aragón y Roma.

1119 Alfonso I durante el papado de Calixto II [1119-1124] (Guido de Borgoña, tio carnal de Alfonso Ramírez) efectúa la restauración de las iglesias de tierras reconquistadas y nombra los obispos: de Tarazona a don Miguel, antiguo monje de San Sernin (Tolosa); de Sigüenza a don Bernardo, también francés, de Segovia a don Pedro, otro clérigo francés. $y$, en Tudela, entonces más importante que Tarazona, establece una Iglesia Mayor con su cabildo dependiente del prelado de Tarazona.

1120 Norberto, arzobispo de Magdeburgo funda la Oden de los Premostratenses para moralizar la vida de los canónigos.

1120 Se difunde la Bula «Etsi Judaei» tratando de detener el furor antijudío.

1123 Fin de la Querella de las Investiduras en IX Concilio de Letrán que estableció la $a b$ soluta independencia de la Iglesia en las elecciones episcopales.

1134 En agosto Alfonso I aún designa obispo para Huesca a Dodon, el hasta entonces abad de San Juan de la Peña; para Roda a su hermano Ramiro y para las diócesis de Huesca y Mequinenza a Fortún Galíndez.

1138 Muere el judío barcelonés Abraham bar Hiyya (Savasorda) que enseñó en diversas ciudades de Castilla y sur de Francia, después de estudiar en Lérida, Zaragoza y Huesca. En sus obras La forma de la tierra y el Cálculo del curso de los astros se exponen por primera vez las ideas de Ptolomeo en hebreo, también hizo unas Tablas astronómicas. Fue traductor del árabe al hebreo.

1139 Muere Avempace.

1139 San Raimundo de Fitero (1090-1163) formado en el monasterio pirenaico de Scala Dei, fundó la primera comunidad del Cister en España, establecida en 1139 en Nioncevas (cerca de Pamplona) y, en 1140, en el monasterio de Fitero.

En 1161, al abandonar los Templarios Calatrava, fundó la Orden de Caballería de Calatrava para hacer frente a los almohades, Orden confirmada por el papa Alejandro III en 1164.

\section{Contexto político y social}

\section{Ibérico-aragonés}

1068 Nace Pedro I. Su padre el rey Sancho Ramírez peregrina a Roma y el Papa le titula Rey de Aragón por la gracia de Dios. Sancho Ramírez, al quedar viudo, se casa con Felicia (nieta del rey de Francia Roberto el Piadoso). Le dio tres hijos: Alfonso, Gonzalo y Ramiro

1073 Nace Alfonso I (sus hermanos: Gonzalo en 1078, y en 1094 Ramiro)

1077 Sancho Ramírez concede una «Carta de libertades» a Jaca fomentando economía y repoblación. Impulsó el románico y estableció una casa de acuñación de monedas de plata

1079 Sancho Ramírez nombra a Esteban, canónigo de Jaca, tutor de Alfonso I

1079 Muere al-Muqtadir. La rivalidad de sus hijos hace vulnerable la taifa.

1084 En Aragón, el judío converso Pedro de Almería, es nombrado por Sancho Ramírez preceptor de su hijo Pedro.

1085 Ahmed II sucede al-Mutamin y sigue con el mismo visir, Abu-l-Fadl-ibn Hasday, que sus dos predecesores. 
1086 Alfonso VI de Castilla conquista Toledo, Invasión almorávide por el emir Yusuf ibn Tashufin, que derrota en Sagrajas (1086) a Alfonso VI pese a la valerosa ayuda de Pedro I, su hermano Alfonso y caballeros francos y normandos.

1094 Pedro I, rey, derrota a Ahmed II en la batalla de Alcoraz. Al-Mustain sucede a Ahmed II.

1094 Pedro I conquista Huesca que hace capital del reino de Aragón. Esteban es nombrado obispo de Huesca

1097 Pedro I y su hermano Alfonso ayudan al Cid a ganar la batalla de Bairen.

1099 Muere El Cid Campeador y el 1102 los almorávides conquistan Valencia.

1104 Pedro I conquista Tamarite y a final de año le sorprende la muerte.

1104 Es coronado Alfonso I rey de Aragón. Su sinceridad religiosa condicionada por el ideal familiar de apertura a Europa y exigencias de la reconquista. El obispo Esteban, su amigo y confidente, peregrina a Jerusalén (1104-1106)).

1106 Muere el jefe almorávide Yusuf ibn Tashufin. La lucha en el valle del Ebro adquiere ideal de Cruzada, y presta apoyo a caballeros que acuden a Tierra Santa.

1108 Los almorávides derrotan en Uclés a Alfonso VI donde muere el infante don Sancho. Su hermana Urraca (viuda con un hijo), al morir Alfonso VI en junio de 1109 sin otro heredero, se casa con Alfonso I en otoño del mismo año. Alfonso I fue así rey de Aragón y de Castilla e «Imperator Hispaniae». Dado el carácter y veleidades de doña Urraca, tuvo multitud de problemas y sinsabores hasta que, cinco años después, la Iglesia anuló el matrimonio.

1108 Muere al-Mustain wali de la taifa de Zaragoza. Le sucede su hijo Imad al-Dawla, pero un error político y pese a la ayuda de Alfonso I propició el que los almorávides ocuparan la ciudad dirigidos por Ibn al-Hayy wali de Valencia.

1115 Muere Ibn al-Hayy en combate y le sucede en Zaragoza Ibn Tifilwit que vivió fastuosamente en la Aljafería con Avempace como visir.

1118 En un concilio en Toulouse, Alfonso I consigue apoyo papal, apoyo militar de caballeros franceses más jinetes y arqueros convocados por el obispo Esteban; el 18/XII se rendía Zaragoza Las capitulaciones fueron prueba de la generosidad y caballerosidad del Rey nombrando «tenente» a Gastón de Bearn

1118 Alfonso I conquista Tudela con igual capitulación que Zaragoza. Y a los judíos les concede el mismo fuero que a los de Nájera. Ocupa Tarazona y restablece la sede episcopal..

1118 Recupera Soria, que repuebla y da fueros.

1122 Corfirmación de la Cofradía de Belchite, como «militia Christi» de la frontera. al modo de las órdenes de Jerusalén.

Alfonso I el Batallador fue un «señor de la guerra» pero con la reconquista debió resolver dos graves problemas: Defensa y repoblación de los nuevos territorios. Nombró tenentes y dio fueros especiales. En Zaragoza se asentaron bearneses y en Tudela normandos. Pero también repobló con mozárabes (como los que trajo de su expedición a Granada).

1126 Muere doña Urraca y su hijo, coronado Alfonso VII rey de Castilla y León, exige a Alfonso I el Batallador la devolución de los territorios de Castilla.

1130 Mueren el obispo Esteban y Gastón de Bearn derrotados por Ali al-Lamtuni.

1130 Alfonso I cuida la repoblación cántabra

1134 Derrotado Alfonso I ante Fraga, muere meses después, dejando el reino a las Órdenes: del Temple, del Hospital y del Santo Sepulcro. Pero «frente a un rey idealista y testarudo, unos vasallos atentos a sus intereses y a los de su reino» hicieron que «Cuando, tres días después, el 7/LX/1134, al morir el rey, su hermano Ramiro fuese reconocido inmediatamente como rey por la nobleza del país» como Ramiro II «el Monje». Éste se casó con Inés de Poitiers, hija de Guillermo IX duque de Aquitania, viuda de Aimeri $\mathrm{V}$, vizconde de Thouars del que había tenido ya tres hijos. 
1134 Ramiro II tuvo una hija, Petronila, y al concertar su matrimonio con el hijo de Ramón Berenguer III el Grande, quien sería Ramón Berenguer IV (1131-1162), al mismo tiempo le encomendó el gobierno del reino en calidad de lugarteniente y príncipe de Aragón.

1145 Los almohades a las órdenes de Abu Yusuf Yaqub invaden al-Andalus, fanáticos integristas obligan a realizar conversiones masivas al islam.

1148 Ramón Berenguer IV reconquista Tortosa.

1149 Ramón Berenguer IV reconquista Lérida, con lo que completó la Reconquista de Cataluña.

1150 Boda de Petronila con Ramón Berenguer IV. Con él y su norma jurídica Usatges de Barcelona. se sentaron las bases de la Corona de Aragón, que su hijo Alfonso II instauró.

\section{Ultrapirenaico}

Con Oton I «el Grande», coronado en Roma por el Papa Emperador del Sacro Imperio Romano, y su Privilegium Othonis se inicia la universalidad del poder secular sobre la Iglesia. Oton III admiró a Gerberto de Aurillac, quien de 967 a 970 estudió en Ripoll [más tarde el papa Silvestre II (999-1003)] y fue pionero en la difusión de la cultura andalusí en Europa, como lo fueron las embajadas de Oton I y Hugo Capeto. Pero ante los nombramientos de papas por Enrique III y la reunión de su sucesor Enrique IV «el Grande» en Tribur con el papa Gregorio VII, comenzó la «Querella de las Investiduras» (1075-1122) entre el Imperio y el Pontificado. El papa excomulgó al Emperador..

1078 Los Turcos conquistan Jerusalén. La secta de los «asesinos» retiene a cristianos y exige rescates.

Hugo de Semur (1049-1109) y Pedro el Venerable (1109-1159) construyen la catedral monástica de Cluny, la mayor iglesia de la cristiandad hasta el siglo XVI en que se construyó la iglesia de San Pedro en Roma. De setenta y tres monjes en 1063 pasó a más de trescientos en 1122 .

1096 Urbano II predicó en Clermont la $1^{\text {a }}$ Cruzada a Tierra Santa. En Francia bajo los Capetos, los Nobles obsesionados por adquirir méritos para la tierra y el cielo, dan libertad a los siervos de la gleba que abandonan el campo y se dedican al comercio y artesanía bajo tutela real, lo que inicia el proceso de la extinción del feudalismo.

1099 Los cruzados conquistan Jerusalén y derrotan al fatimí al-Afdal en la batalla de Ascalón.

1104 En Alemania encarcelan a Enrique IV, engañándole y obligándole a abdicar a favor de su hijo.

1106 El Emperador Enrique V que se impuso al Papa Pascual II. El sucesor de éste, Calixto II, con ayuda francesa, en 1122, hizo firmar a Enrique V el Concordato de Worms. Así se dio fin a la Querella de las Investiduras.

Mientras tanto, Inglaterra, regida desde 1060 por la Dinastía Normanda que introdujo el francés como lengua institucional y con unos señores feudales terratenientes, se consolida como reino bajo el gobierno de Guillermo II el Rojo (1087-1100) que debió hacer frente al duque de Normandía hasta ser asesinado en una cacería sucediéndole su hermano

1100-1135 En Inglaterra Enrique I Beauclerc rehizo la unidad anglonormanda al anexionarse la Normandía y, en el año 1114, promulgó las «Henri law»

1113 Para cuidar a los peregrinos desvalidos o enfermos en Jerusalen se creó la Orden de San Juan del Hospital, más tarde militarizada llamada Rodas y Malta.

1119 Guillermo IX (1071-1124), Duque de Aquitania, participa en la reconquista en Aragón. Antes, en 1101, había participado en la Cruzada a Tierra Santa. Pero sobre todo se le recuerda por ser el más antiguo trovador conocido y gran poeta (aunque de «doble cara»: cínico y cortes, tierno). 
1119 Para defender los santos lugares surge la primera órden de monjes guerreros, la «Orden del Temple». Su primer Gran Maestre fue Hugo de Payns.

Otras órdenes de aquella época son las del «Santo Sepulcro» y la «Orden Teutónica»

1122 En el norte de África se organiza la secta almohade que hizo huir a los judíos hacia la España cristiana y al papa Celestino III urgir la unidad de los cristianos frente el islam.

1130 Cisma en la Iglesia. Se nombran dos papas: Gregorio Papi con el nombre de Inocencio II y a Pedro Pierleone (monje de Cluny e hijo de un judío converso) con el nombre de Anacleto II. San Bernardo pide al Emperador Lotario III del Sacro Imperio Romano que impida ejercer a Anacleto II.

1133 Lotario III es coronado por Inocencio II.

1135-1154 Esteban de Blois sucede en Inglaterra a Enrique I el Clérigo, y deberá hacer frente a un periodo de anarquía entre sus señores feudales con la pérdida de Normandía

1136 Los musulmanes turcomanos se apoderan de Antioquía y Trípoli.

1137 Acaba el cisma por la muerte de Anacleto II. San Bernardo consigue el acuerdo de Inglaterra, Francia y Alemania a favor de Inocencio II.

1150 Surge en centro europa el movimiento Cátaro de los «buenos hombres», que sirvió al rey de Francia para apoderarse del Langudoc y Provence. En la Provenza se van concretando los círculos de estudio sobre la Kábala..

1162 Muere inesperádamente en el Piamonte Ramón Berenguer IV cuando su hijo mayor sólo tenía cinco años. Reunidos los magnates aragoneses y catalanes en Huesca, le rinden honores y se cambia su nombre por el significativo de Alfonso II.

\section{EQUIVALENCIA DEL ENLACE DE ARAGÓN Y EL ICONO DE CHARTRES}

El Centro Escolar Catedralicio de Chartres, en el siglo XIII, publicó un manuscrito con el dibujo denominado por M. Didron ${ }^{3}$ «icono de Chartres», manuscrito que, desgraciadamente en un bombardeo, fue destruido durante la II Guerra Europea. Una de las imágenes de este manuscrito está reproducida en el dibujo de la derecha de la figura 2 . En la monografía $n^{\circ} 18$ de la Academia de Ciencias de Zaragoza (junio 2001) demostrábamos que tal icono se deduce fácilmente de la figura 1 de Pedro Alfonso reproducida en la página 31 . Veámoslo.

En primer lugar, Pedro Alfonso se encontró con los símbolos que de la Trinidad consideraban los cristianos primitivos (el triángulo equilátero) y el de San Agustín (tres anillos iguales de igual substancia). figura 2 a la izquierda. Pedro Alfonso con una adecuada «suma» de los cuatro elementos constitutivos, introdujo el dibujo central de la figura 2 , mientras que a su derecha hemos representado el enlace definido por el icono de Chartres.
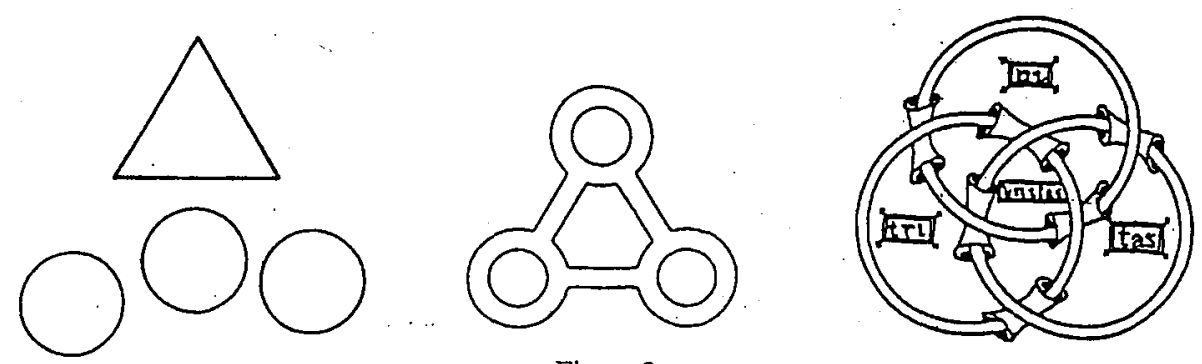

Figura 2

3 M. Didron and A. N. Didron, Christian Iconography, or the History of Christian Art in the Middle Ages, George Bell and Sons, London, 1886. 
Pero a un Pedro Alfonso, profundo conocedor del Sefer Yesirah (con su cosmogonía místico-lingüística que supone que «todo comenzó a existir... por las combinaciones con repetición de las veintidós letras del alfabeto hebreo tomadas de dos en dos») y del Talmud (con la cita a Bezalel, el arquitecto del tabernáculo), no le sería difícil asociar esa unidad de cuatro elementos con el tetras, o tetragramaton, griego de YHWH, el nombre de Dios, y de ello que asociara con cada persona de la Trinidad las tres combinaciones de dos letras tomadas de las tres distintas Y, H, W; o que en el icono de Chartres se descomponga la palabra en las tres sílabas... Pero esto lo realizó Pedro Alfonso. Nuestra exposición trata de hacer ver la equivalencia de la figura del centro (Pedro Alfonso) y la de la derecha (icono de Chartres).

Consideremos la correspondencia biunívoca entre

$$
\text { Triángulo equilátero — Trilátero equiángulo }
$$

Que a un triángulo equilátero $(\mathrm{ABC})$ asocia él mismo como trilátero equiángulo (abc) Es decir a la figura intersección de ángulos asocia la figura intersección de semiplanos

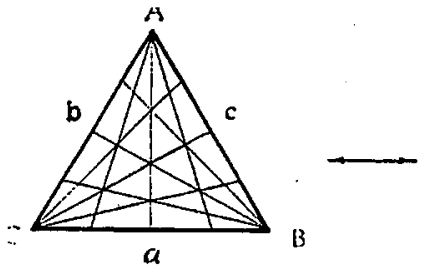

Figura 3

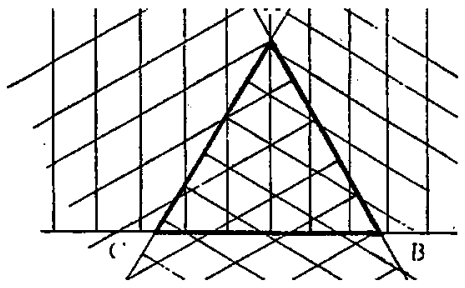

Figura 4

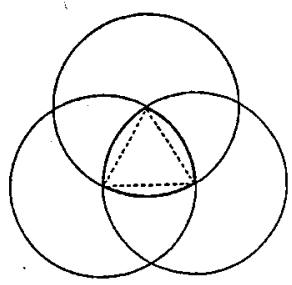

Figura 5

Y si se tiene en cuenta que la compactificación de las rectas soporte de los lados (de los semiplanos del que son bordes) son circunferencias (resp. círculos), la compactificación de la figura 4 viene dada por el dibujo de la figura 5 situada a la derecha. Figura que, trivialmente, nos «esquematiza» el icono de Chartres. Para obtenerlo basta materializar la compactificación realizada operando sobre un trilátero equiángulo de lados trozos de madera, alambre, cuerda, etc. dispuestos como en la figura 6 a la izquierda, donde los vértices se forman al pasar un lado, por ejemplo el «a», (de trazo continuo en el vértice $\mathrm{C}$ ) sobre el otro, el «b», (que aparece como cortado por el «a»). Una cuidadosa pero sencilla distinción de los puntos comunes a una u otra parte de todo el plano respecto a los semiplanos (luego a si pertenecían a uno, dos o tres semiplanos) nos determina la figura 7 más abajo. Como cada uno de los pares de aros aparecen así enlazados por el tercero, queda probado como la construcción de Pedro Alfonso nos determina el enlace o icono de Chartres.

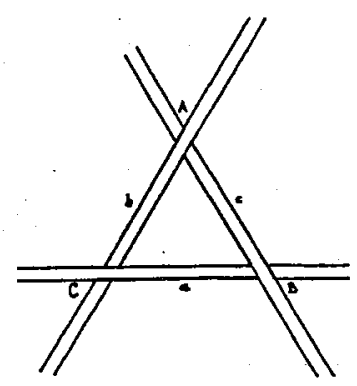

Figura 6

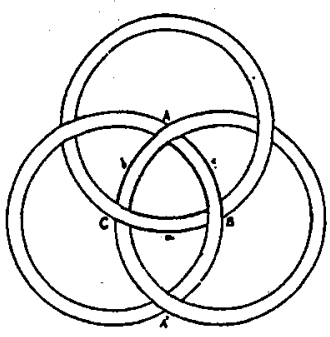

Figura 7 
De hecho, intuitivamente, la correspondencia establecida nos asocia con el símbolo de Pedro Alfonso, obtenido mediante la llamada «cirugía de variedades» en matemáticas, el correspondiente «icono de Chartres» obtenido por pegado a los extremos de los lados del triángulo material los extremos libres de los anillos cortados, cuidando respetar la alternancia de su cruce en los vértices. Como se expresa esquemáticamente en los dibujos siguientes:
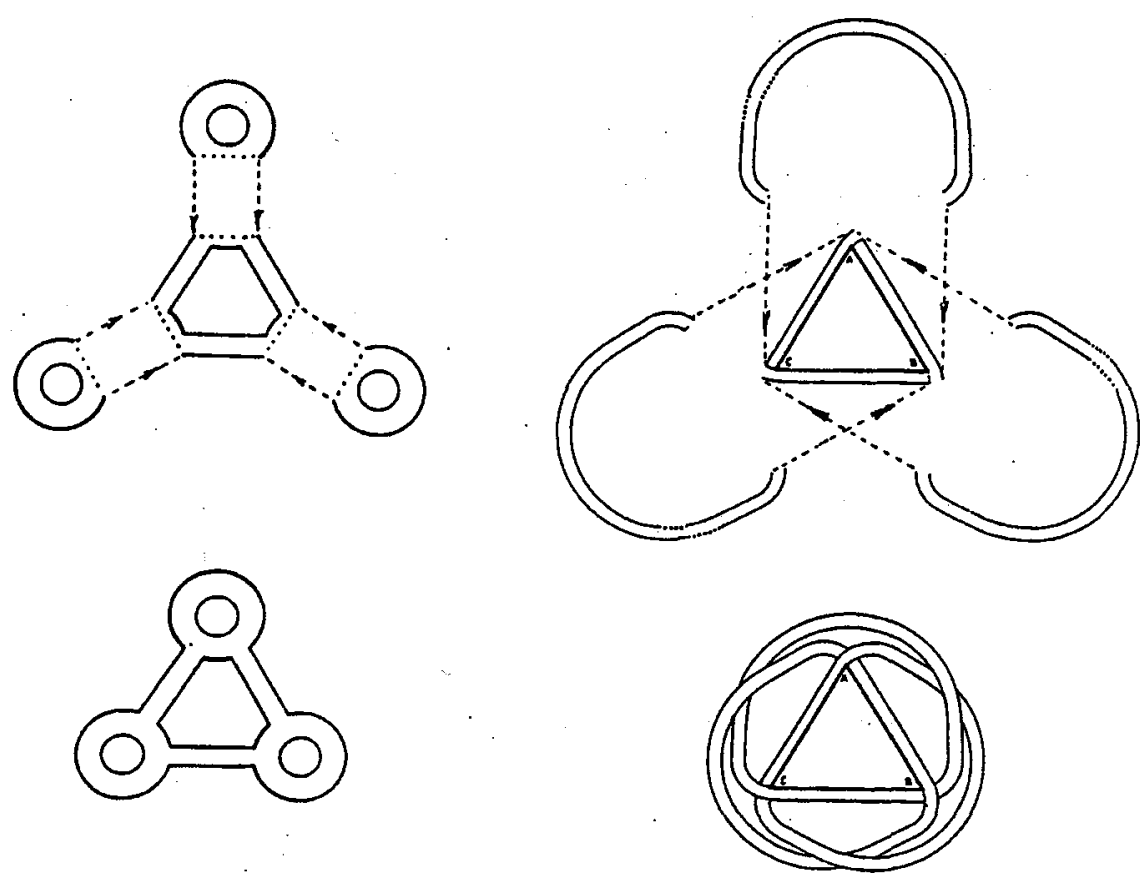

En donde, como de costumbre, los trazos de puntos designan la porción de aro que pasa por debajo de otro aro en la figura final.

Concluiremos esta comunicación con tres aplicaciones: el monedero de malla, la mesa frigia desmontable y una posible verificación experimental relativa al nudo gordiano.

\section{MONEDERO DE MALLA}

El primer ejemplo de materialización del enlace matemático que estamos considerando, distinto del que denominamos «enlace de Aragón», se obtiene fácilmente por reiteración del proceso de construcción de este último con cuerda o alambre. Puesto que hoy nos es más accesible «soldar» con una cerilla los extremos de un trozo de cuerda de nylon que los extremos de un trozo de alambre, consideremos tres trozos de cuerda iguales (o distintos) $a, b, c$ :

\section{1.}

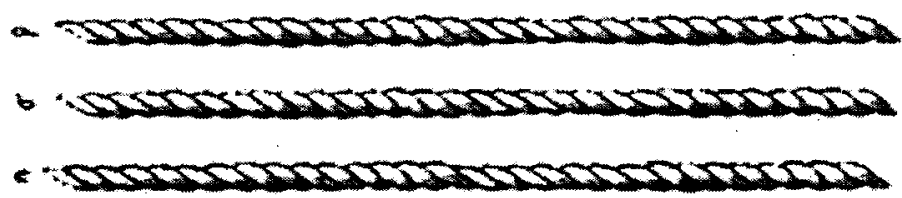


De los trozos $a$ y $b$ se obtienen los aros $a^{\prime}$ y $b^{\prime}$ en la figura $2^{a}$. El $c$ se sitúa según la figura $3^{a}, y$, al unir sus extremos, se obtiene la figura $4^{a}$.
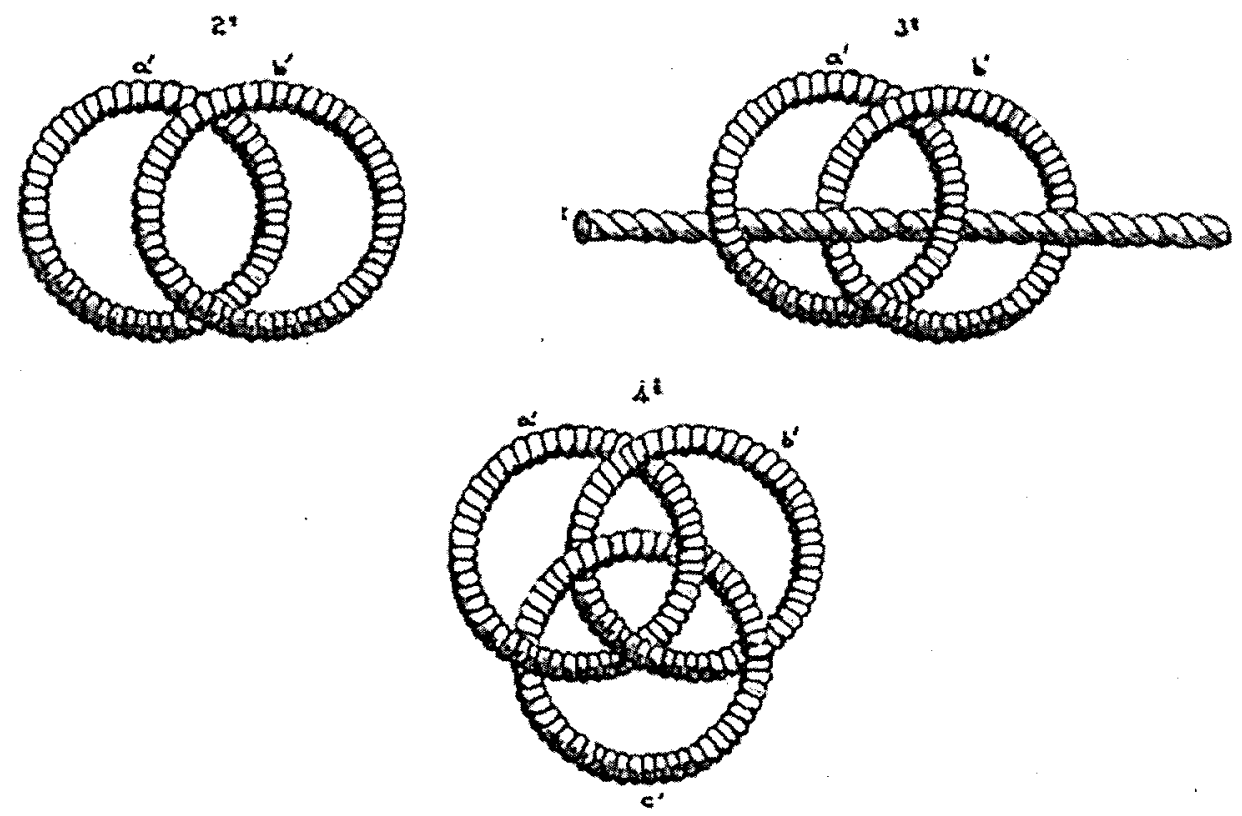

La figura $4^{\mathrm{a}}$ es una materialización análoga a la del «enlace de Aragón». Obsérvese que dos cualesquiera de sus anillos están en posición análoga a los dos de la $2^{\mathrm{a}}$ figura. El proceso indicado por el paso de la $3^{\mathrm{a}}$ a la $4^{\mathrm{a}}$ se puede reiterar indefinidamente, obteniéndose una malla o tejido, con el que se confeccionan diversos objetos, por ejemplo, el análogo al monedero de la izquierda que, realizado en pequeños anillos metálicos (de plata normalmente, aunque también de bronce), fue muy utilizado por nuestros padres y más aún en el siglo XIX.

La segunda materialización del «enlace alternado de tres circunferencias dos a dos no enlazadas» nos lo proporciona una mesa plegable que, últimamente, se puede adquirir en los vendedores ambulantes de origen marroquí, mauritano o senegalés, cuyo origen, como nos precisó el Prof. Pradines de la Universidad de Toulouse, hay que buscarlo en los pueblos nómadas de la meseta de Anatolia y Siria o

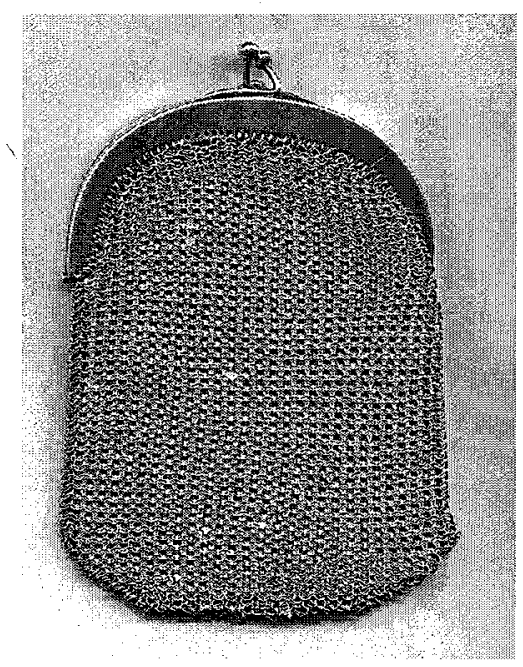
de Mesopotamia. De no ser conocida en la península antes del siglo VIII, se puede suponer que llegó con la invasión árabe y, por lo tanto, también sería conocida por nuestro protagonista Pedro Alfonso. 


\section{MESA DESMONTABLE DE ORIGEN FRIGIO}

Verificación de que el trípode es una materialización como la del enlace alternado de Aragón o Pedro Alfonso.

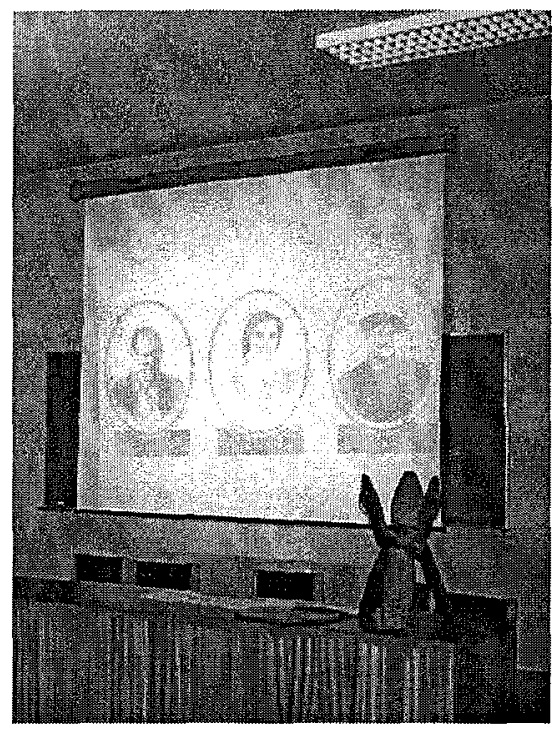

Vista parcial del aula de la Residencia de Jaca (Universidad de Zaragoza) donde tuvo lugar el «IX Encuentro de Topología Algebraica» del 2 al 4 de mayo de $2002 »$.

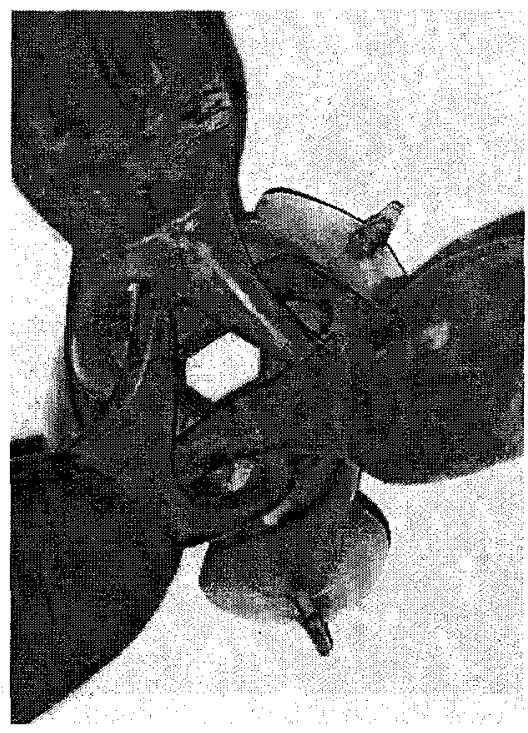

Vista por la parte inferior del trípode mostrando el enlace de Aragón o de Pedro Alfonso.

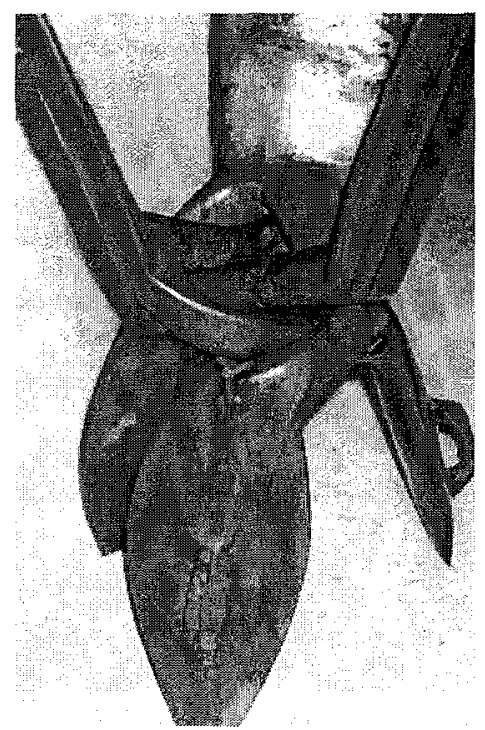

Vista lateral del trípode invertido evidenciando el enlace de Aragón o de Pedro Alfonso.

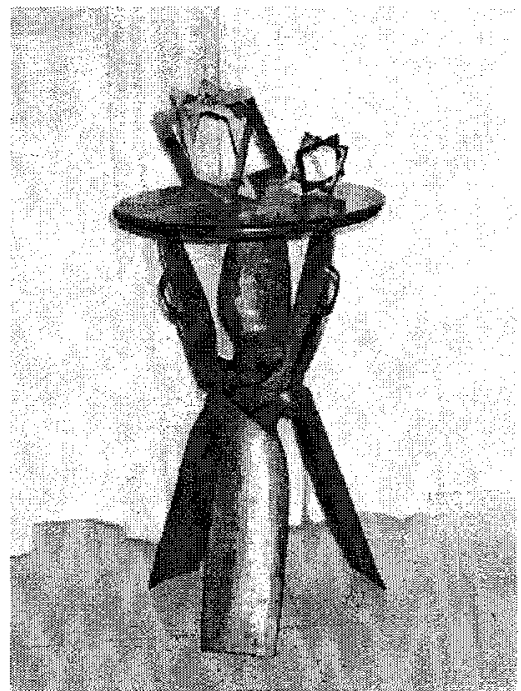

Visión general después de proceder a «montar la mesa» en sentido musulmán (con dos representaciones del enlace de Aragón sobre ella). 


\section{EL NUDO GORDIANO}

La tercera histórica materialización del «enlace alternado de tres anillos dos a dos no enlazados» lo constituye el mítico «nudo gordiano». La primera alusión nos la dio, hacia 1960, un buen amigo de origen hindú profesor en la Universidad de París. Recientemente hemos sabido que un profesor de Ginebra comparte la misma opinión. Este verano intentamos verificar tales afirmaciones. Tomamos un antiguo yugo de una casa de campo del Pirineo y, con la reproducción por un carpintero del extremo del timón de un carro (incluida la clavija), verificamos que, efectivamente, nuestra materialización del citado enlace con cuerda de nylon, sujetaba perfecta e inseparablemente (salvo por rotura) el yugo al timón del carro. Ver las fotografías más abajo.

Aunque quizás el origen se halle en el Viejo Reino Hitita (1800 a. C. en la región de Anatolia y Siria), la historia lo refiere al reino de Frigia con capital Gordium (de máximo esplendor en los siglo IX y VIII a. C.) Parece ser que el carro de su rey, Gordio (o Midas), tenía el yugo sujeto al timón mediante el denominado «nudo gordiano». Nudo hecho de tal modo que no era posible encontrar sus extremos. Cuenta la leyenda que un oráculo había prometido el dominio de Asia al que fuera capaz de desatarlo y que, hacia el 330 a. C., cuando llegó Alejandro Magno a Gordium, en lugar de desatarlo, lo cortó con su espada. Leyenda que «alude a un problema que sólo puede resolverse por una acción drástica».

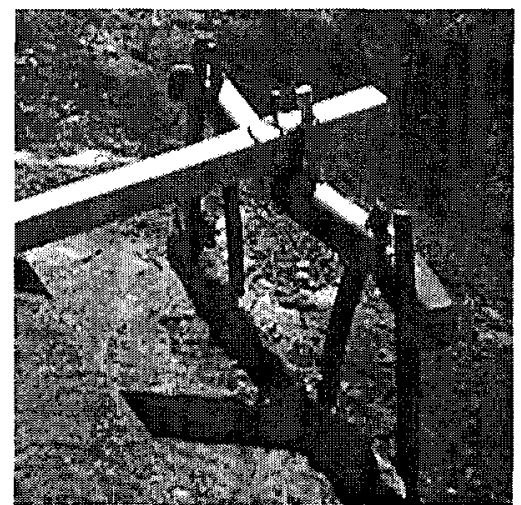

Vista posterior derecha

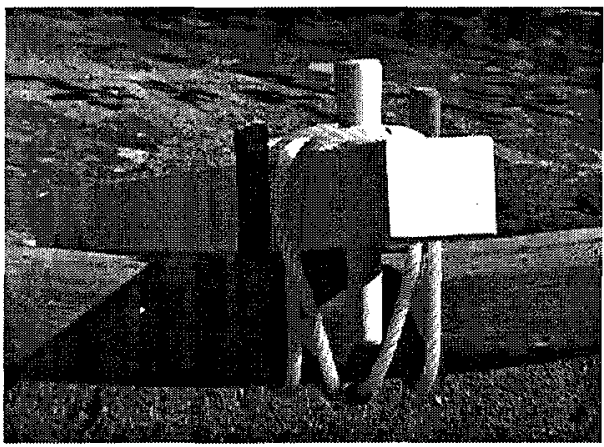

Vista frontal del nudo

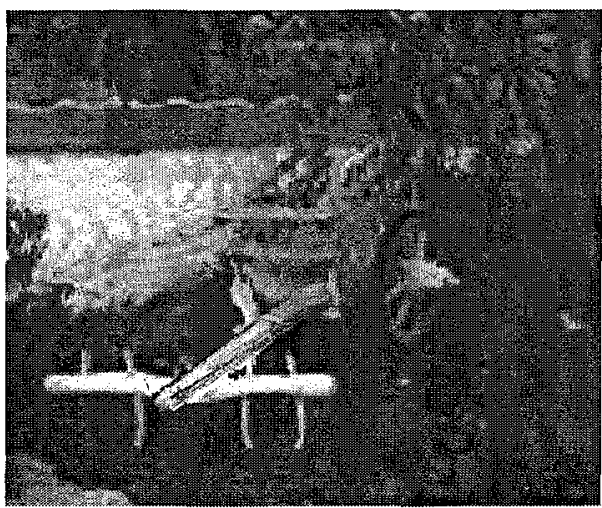

Carreta del Pirineo con yugo análogo al utilizado

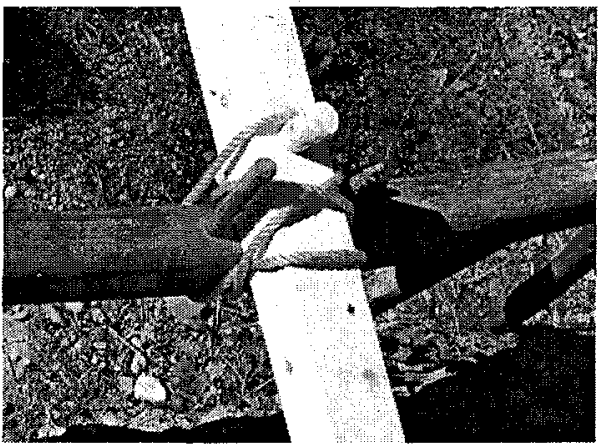

Vista cenital posterior izquierda 


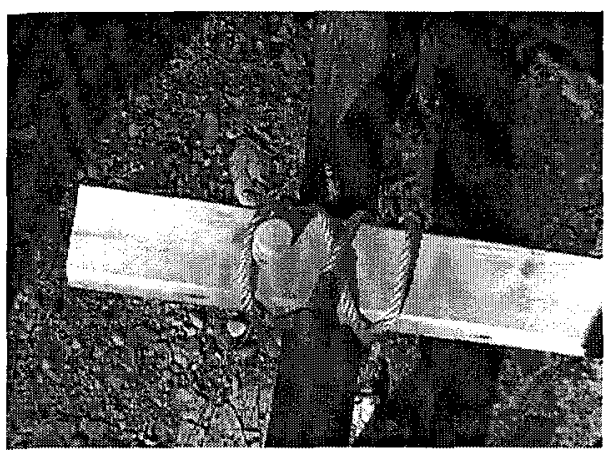

Vista cenital lateral izquierda

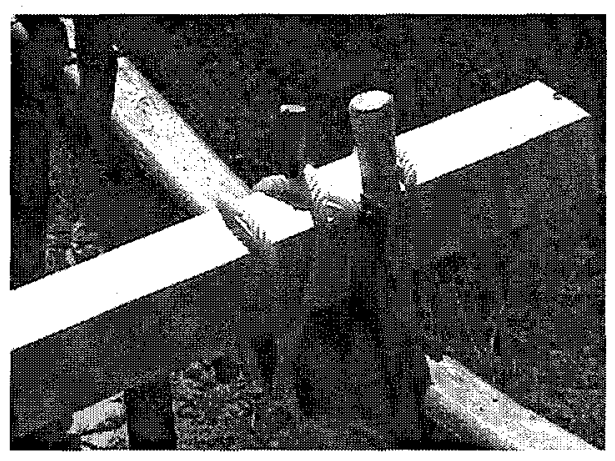

Vista lateral derecha

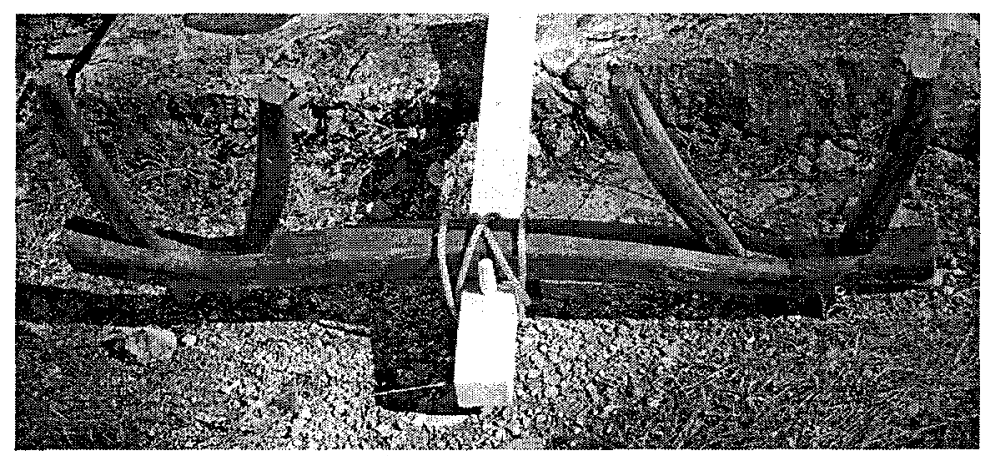

Vista inferior del yugo atado al timón

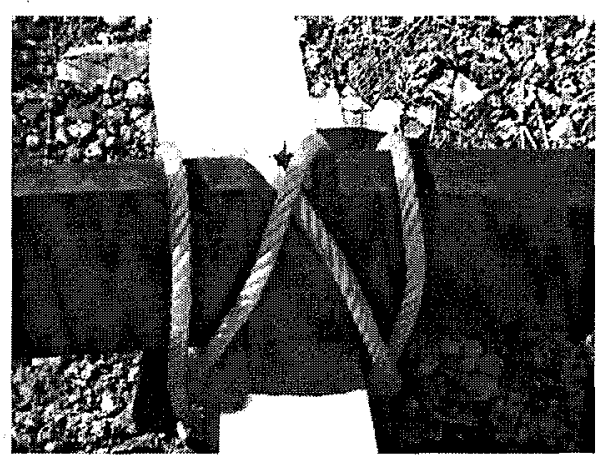

Detalle de la parte inferior del nudo

José L. Viviente Mateu Profesor Emérito

Facultad de Ciencias e-mail viviente@unizar.es 